\title{
Designing Social Networking Sites for Older Adults
}

\author{
Lorna Gibson \\ School of Computing \\ University of Dundee \\ lgibson@computing.dundee.ac.uk \\ John Arnott \\ School of Computing \\ University of Dundee \\ jarnott@computing.dundee.ac.uk
}

\author{
Wendy Moncur \\ Department of Computer Science \\ University of Aberdeen \\ wmoncur@abdn.acuk \\ Christopher Martin \\ School of Computing \\ University of Dundee \\ cjmartin@computing.dundee.ac.uk
}

\author{
Paula Forbes \\ School of Computing \\ University of Dundee \\ pforbes@computing.dundee.ac.uk \\ Amritpal S Bhachu \\ School of Computing \\ University of Dundee \\ abhachu@computing.dundee.ac.uk
}

\begin{abstract}
The importance of older adults' social networks in providing practical, emotional and informational support is well documented. In this paper, we reflect on the personal social networks of older adults, and the shortcomings of existing online Social Networking Sites (SNSs) in supporting their needs. We report findings from ethnographic interviews, focus groups and hands-on demonstrations with older adults, where we find key themes affecting adoption of SNSs. We then consider design aspects that should be taken into account for future SNSs, if they are to meet the preferences of older users.
\end{abstract}

Personal social networks, older adults, social networking sites, privacy, communication.

\section{INTRODUCTION}

Friends and relatives are good for us. The practical, emotional and informational support ("social support") that we exchange with them, and the companionship that we share, have beneficial effects including increased longevity and improved mental and physical health [10]. For example, patients who have suffered a stroke or heart attack but had a good amount of emotional support experienced a higher survival rate than those without such support [13]. For older adults, the significance of maintaining diverse social networks is pronounced, as it staves off cognitive decline, depression and isolation. Frequent social interactions create an increased sense of well-being [6]. Therefore, how to increase older adults' opportunities for social inclusion is an important research question [33]. Younger adults are increasingly using online social networking sites (SNSs) to mobilise network capital (the practical, emotional and informational support available within their social network) through sites such as FaceBook. They are able to communicate with a wide circle of friends and relatives (their "social network") cheaply and efficiently, sharing personal information, accessing support, and participating in the wider online community. SNSs also have the potential to assist older adults, in maintaining relationships, yet uptake by older adults is extremely low. Even SNSs developed specifically for them have not achieved popularity with their target user group. With the predicted expansion of the older population, we believe that it is timely to consider how SNSs could help older adults to maintain their social networks, and leverage embedded network capital. With this goal, we first consider the characteristics of older adults' social networks. We then describe the results of studies that we have conducted with older adults. Finally, we propose some design implications for these SNSs and thoughts on future research directions. Research at the School of Computing is ideally positioned to do this. It houses a unique space - the User Centre - where older adults and technology come together for the benefit of new learning opportunities, social interaction and research [11]. Recent research activities conducted there, related to social networks and social networking sites have been brought together in this paper to try to map the future and needed work on SNSs. The work reported here contributes to existing discussions in the $\mathrm{HCl}$ Community about how to create SNSs that engage older adults. SNSs offer users the opportunity to maintain and extend their social network, thus increasing access to beneficial social support. Our participants were different to those involved in previous studies [such as 18, 31] in two ways: they were familiar with computers and the Internet, and they were in a broader age range (63-86 years) than previously considered.

\section{BACKGROUND}

\subsection{Personal Social Networks of Older Adults}

The size of an individual's personal social network has finite, cognitively constrained limits that transcend cultural and socio•economic groups [29]. Within 
these finite limits, network size and heterogeneity is influenced by personality traits, stage of life and opportunity. Whilst personality is usually stable over adult life, stage of life and opportunity change. This is true for young and old.

\subsubsection{Stage of life}

Social networks go through periods of change relating to life 'transitions', both in terms of the people in the network and the importance of those people. For older adults in particular, network size and membership may be reduced by 'transitions' such as divorce, spousal illness/death, and geographic displacement - either to be closer to family or to move to sheltered housing or a nursing home. Retirement also has strong effects. The transition between work and retirement may influence older adults' perceptions of their social network. Women in particular alter their perceptions of previous colleagues at this time, often re-imagining work relationships as friendships, thus altering their perceived network membership and tie strengths [12].

\subsubsection{Opportunity}

Work is likely to have taken up a large part of older adults' time, and to have played the largest part in the development of their social networks, beyond the inclusion of family members. There may have been little opportunity or funds for additional external socialising. Those doing shift-work may have had their social lives and work-based social interactions further constrained [20]. Workers that spend long periods away from home, such as naval personnel, may experience similar limitations on developing their networks, but tend to have intense friendships based on camaraderie, although not necessarily emotional support. The social networks of older people (usually women) who did not work outside of the home after marriage may display lower heterogeneity through lack of opportunity to meet a wide range of people: the importance of the local community and neighbours is likely to be stronger for them. Once retired, older adults may find it hard to maintain or increase membership of their network. Reduced mobility may make it difficult to meet people [23]. Disabilities common to older people, such as hearing loss and limited mobility, can restrict the ability to communicate - whether face-to-face, or via mediated communications such as the telephone, letters, or emails. Reduced income may also be a constraint. Beyond this, for those experiencing difficult circumstances, sharing of personal information is also governed by the desire for privacy, and a wish not to worry loved ones unnecessarily [22].

\subsection{Social Networking Sites and Older People}

Getting online can have positive benefits for older people. Over two thirds of 'silver surfers' say that using the Internet has improved their lives [1]. Whether it's using FaceBook to stay in contact, Skype calls to family abroad, or blogging to have their say, social networking can empower older people to stay connected and engaged. The possibilities for social interaction by older adults via the Internet can have inspiring outcomes [14]. Yet the uptake of social networking services by older adults is extremely limited. In the UK, $3 \%$ of adults aged 65-74 have used an online social networking service. Uptake for those over 75 is below 1\% [25]. This contrasts strongly with the $35 \%$ of those aged 35-54 years who now use SNSs. Older adults tend to see the Internet as a tool to achieve functional goals such as bill payment, and are ambivalent over its usefulness to them as an information channel for social interaction [31]. There have been attempts to provide social networking sites designed specifically for older adults (such as iYomu), although without significant success. In the UK, Saga Zone provides a social network for over 50s [30]. However, such SNSs exclude younger people who the older adults may want to connect to [2]. It is often younger people who promote/encourage technology use in older adults in the first place [31]. Staying connected with geographically remote grandchildren is a major motivation for older adults in using technology (such as email, web cams and Skype) [17]. An ageinclusive approach may be more appropriate than an age-exclusive one in the long-term. Current SNS designs largely overlook older adults' needs, which differ from those of the young adults for whom SNSs were originally designed [17], [26]. There remains a huge potential for a social networking tool that matches older adults' expectations and preferences. Lehtinen el al. previously reported on SNS use by older adults and on those within the window of retirement (aged 55-65) [17]. Those participants were asked to use an SNS for a period of four weeks. The research presented here differs from this by looking at the interest and feelings related to adopting or trying out SNSs. To facilitate this, we included a range of participants aged from 63 to 86 years old.

\section{METHODOLOGY}

Our approach to this topic is two-pronged: firstly to develop further understanding of the social networks of older adults through focus groups and ethnographic interviews; and secondly to explore the understanding and feelings of older users of technology towards SNSs through focus groups, demonstrations and hands-on experience. The majority of the research was conducted with older adults from the University of Dundee's User Centre. There are two types of participant at the User Centre: 
those that come in briefly to try out new technology or learn how to achieve a specific task; and those who attend regularly and enjoy continued learning opportunities. A regular group of patrons attend the space and run an informal computing club. Apart from the ethnographic interviews, all participants for the studies reported here were recruited from the User Centre. As such we recruited participants who were current Internet users (from the regular attendees of the User Centre) but were not current users of SNSs. The scope of this work was to look beyond the digital divide, and the existing research that demonstrates the reservations older adults have with trying and adopting new technology: much research already exists in that field. Instead we concentrated on investigating the views of those who were already engaged with technology. Understanding their perspective is crucial in determining an appropriate approach to allow SNSs to tap into the older adult market. With active users, it is possible to discount typical hurdles such as difficulties using a computer/mouse or lack of understanding of software conventions. Problems or lack of interest encountered by active users are more likely to be due to issues with appeal, presentation or application of the SNSs. The results presented in this paper should provide further insights into what older adults want from SNSs.

\subsection{Focus Groups}

To study older adults' social networks and to explore their thoughts and feelings towards SNSs, we conducted two focus groups (in total, $n=17$; female $=12$, male $=5$; age range 63-86). Participants were recruited from the User Centre group and were all current users of the Internet who had never used a social networking site. The two focus groups were drawn from a socio economically diverse group with DepCat scores from 1-5 [21]. Each focus group lasted 1.5 hours and covered the following topics:

\section{- Who is in their social network?}

- What mechanism do they use to communicate with social network members, and how often?

- What is their understanding of SNSs and what do they base this opinion on?

- What do they expect to be able to do on a SNS?

- What benefits and concerns do they have with SNSs?

\subsection{Ethnographic Interviews}

Ethnographic interviews were conducted with older adults $[n=4$, male $=2$, female $=2]$ who had recently moved into sheltered housing. The aim of the interviews was to get to know the participants and their network of support. The interviews were conducted in the participants' homes, and were informal conversations lasting on average an hour. This allowed exploration of the social networks of those in a transitional period, specifically those moving from independent living to assisted living.

\subsection{Demonstrations}

A series of demonstrations of social networking sites (MyFriendsOnline and FaceBook) took place in order to facilitate further exploration $(n=15$; female=8, male=7; age=63-81). This group was drawn from a socio-economically diverse group with DepCat scores from 1-5 [21]. The demonstrations involved both an overview presentation of the key features (such as finding friends, posting messages and locating relevant interest groups) as well as a hands-on session where participants could explore and try out some of the features themselves. Many of the participants felt uncomfortable $(n=9)$ having to register with the SNS in order to explore or 'play around' in it, making it very difficult for them to get a handle on how the sites worked. In these instances, they were given an opportunity to use dummy accounts to evaluate the site but we note that richness of the experience was affected by prevalence of fake information in the dummy accounts. However, it did provide these participants with an experience of SNSs that was not otherwise available to them due to their unwillingness to register their details.

\subsection{Follow up focus group}

A follow up focus group took place 8 months after the first focus groups $(n=10$; female $=7$, male $=3$; age $=63$ 79). Participants had also taken part in the previous focus groups and demonstrations described above, and were a socio-economically diverse group with DepCat scores from 1-5 [21]. During this focus group, a summary of previous thoughts and findings were presented followed by an open discussion on:

- The findings from the previous focus groups

- Current feelings towards SNSs

- Their experiences of SNSs in the intervening period. 


\subsection{Data Collection and Analysis}

During all the studies, a rich collection of videos, ethnographic observations and researcher notes were gathered. Data was analysed using thematic analysis [4]. This method allows specific experiences and anecdotes to be grouped together by similar attributes and then eventually as overarching themes. It is particularly useful for drawing messages from rich datasets provided by the focus group method and ethnographic interviews. The insights were synthesized into the findings presented here on the offline and online social networks of older adults.

\section{INSIGHTS INTO OFFLINE SOCIALNETWORKS}

\subsection{Results of focus groups}

Results from the ethnographic interviews and the first set of focus groups provided insights into older adults' offline social networks. Overall, the participants reported that their social networks consist largely of relatives (of varying degrees of closeness), friends acquired prior to retirement from work or their previous social life, and new friends acquired during retirement (often through shared social activity or shared experiences such as moving to a sheltered housing complex). One key way in which their social networks changed was through death of a network member:

"My social network is changing. People are 'dropping off the perch' -my network is different now." (Focus group participant)

Contrary to their expectations, participants' relationships with relatives did not provide the "bread and butter" relationships that they could count on for emotional and practical support during retirement. Instead, participants described how they "check in with (relatives) every week", typically by telephone. Distance played a part in this: participants were often separated from their relatives by either physical or emotional distance. For example, emotional distance could arise when family members lived nearby but had hectic work schedules and social lives. When older adults relocate (into assisted living or to be nearer family), further problems were encountered.

\subsection{Results of ethnographic interviews}

The residents of sheltered housing who were interviewed in the ethnographic study had recently been re-housed and were going through one of the major transitional stages outlined earlier in the paper. Similar to the focus group, the transition caused a physical distance which placed strain on parts of their social network. Maintaining connections with previous neighbors was highlighted as a difficult but desired process. For example, one case reported being dependent on a 'proxy' (the daughter of a neighbor) in order to continue social contact. All participants interviewed commented on the people that surround and support them and with whom they interact daily, weekly, monthly and yearly. Figure 1 depicts a general model that emerged with four notable groups of stakeholders: -formal carers (care professionals) -informal carers -family -professional bodies This model of stakeholders emerged from the interviews, however the network they formed (categorised by geographic distance, frequency of visits and mode of communication) varied. This would suggest that although these older adults' networks contain similar types of stakeholders, the relationships that connect them are personal and individual.

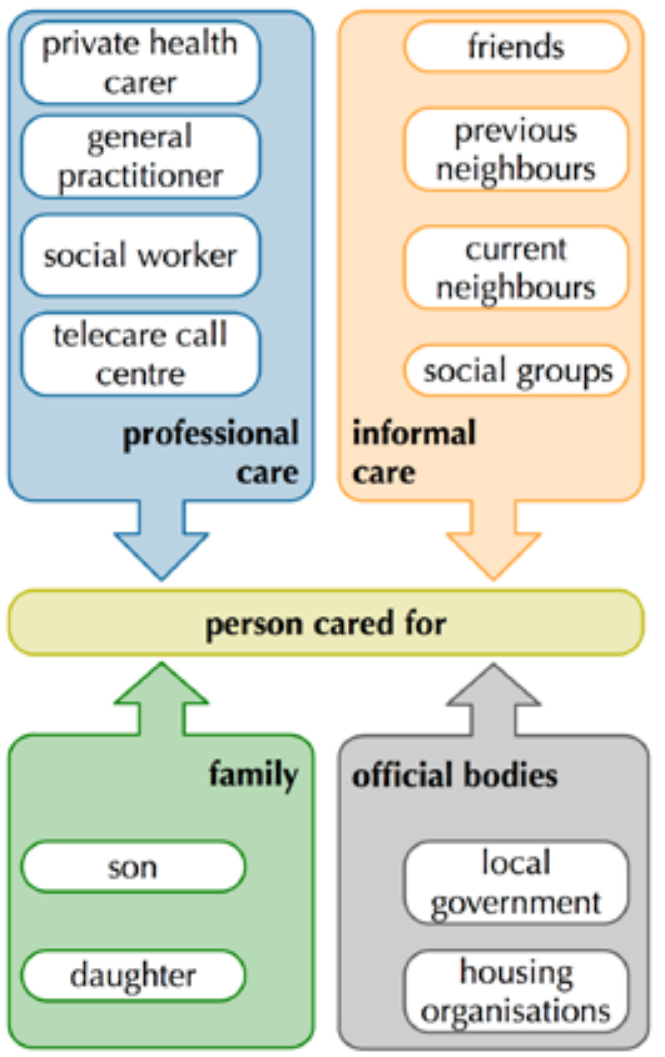

Figure 1 -Model of stakeholder current relationships emerging from the participants in sheltered housing

It should be emphasized that Figure 1 represents the participants from the ethnographic interviews and consequently living in sheltered accommodation. As such their care needs tend to be much higher than those participants from the User Centre. In short, Figure 1 does not present the social network for all older adults but a subset that require additional support from care professionals and informal carers. 


\section{INSIGHTS INTO ONLINE SOCIAL NETWORKS}

Results in this section are presented under the separate research activities conducted: focus groups, demonstrations of SNSs and a follow-up focus group.

\subsection{Focus Groups}

The most immediate finding from the focus groups was the impact that media coverage on SNSs has had on the participants. They were aware of adverse media stories on SNSs, specifically citing young people advertising a party online then having lots of people (not friends) arriving and damaging their house, resulting in extensive damage. In addition, the media generated some misconceptions of what happens on an SNS. For example, they believed that all material posted on an SNS was publicly available on the Internet. They had little understanding that privacy controls exist. The purpose of SNSs was difficult for older participants to grasp. They saw SNSs as most suitable for younger people. In considering which older adults would use an SNS, they suggested that it would be relevant for areas of low population density, where it is hard for people to have physical contact, or for lonely people (perhaps a recluse). Nearing the end of the discussions, some participants reflected that SNSs could provide a useful resource for checking in with widespread family, and with people that they see infrequently. For them, the appeal lay in being able to contact multiple people simultaneously, thus saving repetition. They compared it to a "round robin" letter that they might put in a Christmas card. SNSs aroused privacy concerns. The prospect of sharing personal information on an SNS made participants feel "vulnerable", "scared" and "worried". This was largely down to high profile, continued media coverage of online identity theft. They also believed that there are age-related differences in perspectives on privacy and disclosure: "Confidentiality is totally different for them ...". This is similar to previous research which found older adults did not appreciate publicity as much as they suspected young people did [17]. As stated earlier, most participants did not realize that privacy settings were available on most SNSs. This realisation was reassuring to them, mainly because they felt a little happier about their grandchildrens' use of social networking sites and that "they won't be damaging their reputation". Gender was also identified by older males as a factor in the motivation to use SNSs:

\footnotetext{
“... this social networking is a female thing. I don't speak for long on the phone, but my wife does... social networking is just gossip, isn't it?" (Focus group participant)
}

Finally, the participants also identified the importance and varying degrees of reprocity, similar to Lindley et al., [18].

\subsection{Demonstrations of SNSs}

Demonstrations of an SNS designed for older adults, MyFriendsOnline, generated extensive discussion amongst participants, but no longer-term use. This is despite participants perceiving the site as fun to use during the demonstration. Similar to previous findings in the focus group, participants found it difficult to come to terms with the purpose or focus of the SNS. A common sentiment was "I really can't see where I can fit into this". Many participants felt that SNSs were irrelevant for people that they felt close to, and that it was a stilted communication channel. They found their lack of a clear idea of objectives in using such a site awkward. Their other online experiences to date had a clear purpose -such as using a searching engine to find information or using email to communicate with someone. Moreover, they felt that without a clear grip on the purpose of SNS, they would not know if they were using it appropriately. This concern was amplified when they considered that their SNS use would be conducted in a public arena, where they could potentially embarrass themselves in front of those in their network.

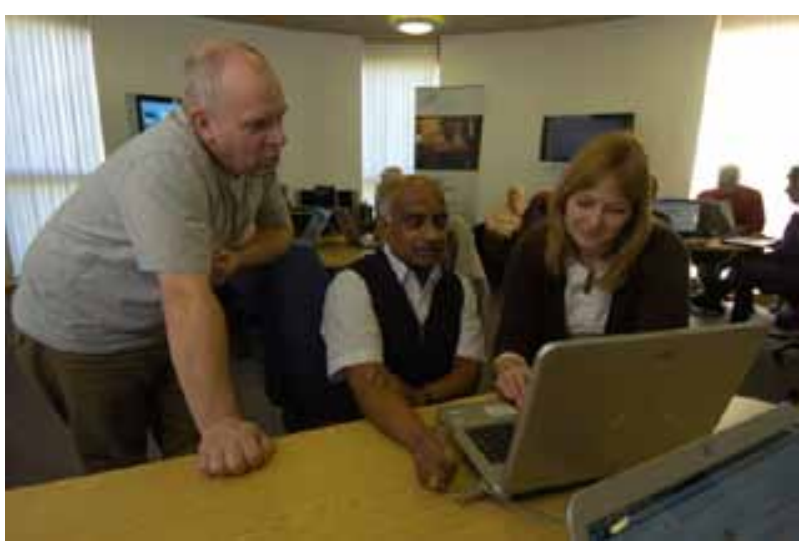

Figure 2 - Demonstration session of social networking sites at the User Centre at School of Computing

Another major problem was the use of the word friends. This word seemed to mean something very important and specific to the older participants. They were very unhappy with the idea that it was used as a catch-all term for anyone from "best friend for years" to "passing acquaintance". As the demonstration took place within the context of the computer club, a few tried to explain (with much embarrassment) that the user group member who they were sitting with was an acquaintance, and that this relationship was very different to that of being a friend. The SNSs that were demonstrated did not contain the kinds of groupings (such as family, close friends, work friends) that they hoped. They found 
this disappointing. The customary binary or tertiary privacy settings (friend/ friend of a friend/ everyone) were particularly disliked. The use of appropriate friendship groupings was identified as particularly important, for example:

"I don't want to share my holiday snaps with every Tom, Dick or Harry".

This interest in maintaining their privacy and distinguishing between what they want to share with family, close friends or acquaintances was identified as very important. Privacy settings have come under much public scrutiny in recent times. An acknowledged problem lies in the counter-intuitive nature of default settings, where all information is categorised as being publicly available. Taking the step of changing these default settings may not be immediately obvious -to younger as well as older adults. There was a reluctance to risk overdisclosure of personal information -

"I don't want to tell them everything about me"

- and a wariness of feeling "on display". One factor that was displeasing to most of the participants was the all-or-nothing approach to group membership. Rather than disclosing their full profile to a group that they had just joined, their preference was to be able to remain on the outskirts and observe. When they felt comfortable sharing personal information, they could opt to reveal more information and become a more active contributor. One participant highlighted this concern by saying:

"I would never walk into a room full of strangers and say ' $\mathrm{Hi}$, my name is John. I am 72 years old. I live in Dundee with my wife and I enjoy listening to classical music.' So why would I want to do this online?" (Focus group participant)

Overall, they felt more secure with a group identity (and the related partial anonymity) when exploring and experimenting, rather than highlighting themselves as an individual. Participants also identified that SNSs do not represent a typical model of dialogue, specifically where there is an opportunity to do a face to face conversation off-therecord, for example if they were sharing a secret. The persistence of interactions via SNSs, long after the event was also concerning for them [8]. This is similar to previous research where older adults worry about committing accidental social blunders [17].

\subsection{Follow up Focus Group}

Eight months after the initial focus groups and demonstrations, we ran a follow-up focus group in order to reflect on our findings with the older adults. Following the initial research, two of the participants had registered to use FaceBook. Both had avoided completing all the profile information, and had only connected with a handful of individuals online. Neither considered themselves as active, regular users; one logged into the site from time to time to "have a wee look" and the other one had been unable to find a way to remove themselves from the site. Since taking part in the first focus groups, the participants noted that they have become even more conscious of press coverage of SNSs, making a specific effort to read related articles. Most recently they have heard about a web site called PleaseRobMe [27] which they believed was meant to help burglars identify empty houses by using the status updates on various SNSs. It was in fact a spoof site to highlight insecure online behavior, but was reported in the press as having malign intent. Unsurprisingly, this provides them with much concern. While the aim of the PleaseRobMe social experiment is to increase awareness of privacy issues, it seemed to have a secondary effect of causing further concern and apprehension to our participants. Finally, a few of the participants reflected that use of SNSs is less about age and more about personality. They felt that SNSs were for extroverts, and that the reason it is more popular among younger people was that in general they were more extroverted. This is consistent with observations from other studies [16], yet does not match research-based understanding of extraversion [3]. 5.3.1 Using Ning It should be noted that during the intervening period between the two sets of focus groups that the User Centre computer club developed their own 'social network site' using Ning [24], an open source social platform. Ning allows people to create their own, tailored social network for a specific population or topic, in their case for their computer club. Although the majority of the patrons do not consider it to be an SNS, it does contain many of the features prevalent in an SNS. The primary purpose of the site is to provide information on upcoming events and classes, share interesting resources, and provide a mechanism to contact other members without having to share email addresses. The resource has proved popular with the group and has built up suitable critical mass ( $76 \%$ of the current membership are active users). The reasons they use to explain this is that the resource has a clear purpose (they have a reason for visiting the site), and that the focus is on the group not the individuals. As the web site is only for the group and not publicly available means that participants feel more secure about sharing information, although most have not posted a photo of themselves (but it is suspected that this is through lack of knowledge of how to do this than a reluctance to do so).

\section{DISCUSSION}

Older users were more cautious in their use of 
SNSs. They were anxious to retain their privacy, and afraid of identity theft. They were concerned at the idea of having to divulge their full details to new online groups that they join. Instead they would prefer a more tentative, incremental approach to revealing their identity. As with Lehtinen et al., the older participants felt that SNSs were forums to seek attention and publicity [17]. This need for privacy, and the desire to share information selectively, has parallels with the offline world. It is consistent with the findings of the evolutionary anthropologist Robin Dunbar and his colleagues. Humans exhibit specieswide characteristics when it comes to the size of their social networks, and their information sharing behavior [15]. They share very detailed personal information with a few stalwart supporters (the support clique), slightly less information with those that they are in frequent contact with, but would not turn to first in a crisis (the sympathy group), and even less with those that they see infrequently and do not feel emotionally close to (the clan) [32]. Given such species-wide characteristics, it makes sense that the personal social networks of older adults have much in common with those of younger people: there are communication challenges posed by geographic separation and busy lives, and a need to stay in touch with network members to varying degrees of frequency and intimacy. Although there is commonality offline, it seems that this is not immediately transferable to online social networks or SNSs. With the older population it can always be argued that there could be technology issues at play, however the majority of the participants in the studies reported here are comfortable and familiar with technology. Therefore, if they are struggling to embrace SNSs then there is a need to understand why. The fact that their own Ning group web site has become so popular suggests that SNSs can be more universally acceptable to older adults in the right context. While purpose was an important theme identified in our work, there is also a danger in trying to place a heavy emphasis on a purpose for an SNS, when it is possible that older people could be as frivolous as younger people within the right setting and with the right design. The uptake of the Ning web site will allow us to compare our users against previous research with existing (often younger) users. For example, we hope to investigate the use of SNSs in strengthening pre-existing offline relationships, and in developing new relationships, particularly during transitional times such as retirement [9]. Whilst conducting such investigations, it is imperative to understand the feelings and experience of older adults. This can lead to the creation of Internet access methods that are strongly preferred to mainstream ones [7].

\section{DESIGN IMPLICATIONS}

In future work at the User Centre in Dundee, the authors hope to explore three emerging areas further with our socio-economically diverse group of technology-savvy older users. These are:

- Investigate approaches to quantifying the strength of network ties, maintaining appropriate levels of privacy and allowing the sharing of information to various degrees of friendship.

- Investigate an incremental approach to revealing personal information which provides a more palatable and friendly introduction for many older users.

Some of the points above may be relevant to the population as a whole and not restricted to older people. A further area that the authors will focus on is the change in social network membership as autonomy declines. There were noticeable differences between the social networks of the autonomous, active older adults who attended the User Centre and those with reduced autonomy who lived in sheltered housing and were reliant on carers to some degree. For these individuals, the informal, horizontal ties with friends and neighbours imbued with mutual reciprocity declined. Formal, vertical ties with healthcare professionals, social services and paid carers increased, with the older adult acting as a passive recipient of institutional support. The authors believe that SNSs could play a valuable role in maintaining the beneficial existing social networks of older adults in the face of a decline of autonomy, caused by physical or cognitive decline. A more difficult area to explore is the matter of designing SNSs to cope with the death of network members. As one of our participants pointed out, social networks of older adults are more prone to change through mortality than those of younger adults. This is an open question [19], which we are excited to explore further.

\section{CONCLUSIONS}

This paper aimed to enhance current research on older adults' and SNSs by providing insights into perceptions of SNSs by older, technology-savvy users. The authors have used these insights to start to consider design implications for SNSs aiming to attract older adults. Further work in this area is both relevant and much needed. This work has provided stimulus for future work which we are currently undertaking. 


\section{ACKNOWLEDGMENTS}

The authors thank the participants who helped with this research and also the School of Computing at the University of Dundee for continued support of the User Centre facility which contributed significantly to the research. The authors acknowledge support from several sources, including UK EPSRC project EP/D049520/1, UK EPSRC project EP/ G002118/1, RCUK Digital Economy Research Hub EP/G066019/1 "SIDE: Social Inclusion through the Digital Economy", UK DTI project "SAPHE: Smart \& Aware Pervasive Healthcare Environment", Scottish Funding Council (SFC) project HR04016 "MATCH: Mobilising Advanced Technologies for Care at Home" and a UK EPSRC Doctoral Training Grant. The interviews of older people in sheltered housing were conducted by Dr. M. McGee-Lennon (MATCH Project \& Glasgow University). The authors are members of the Joint Research Institute for Computational Systems of the SFC Northern Research Partnership (NRP).

\section{REFERENCES}

[1] Age Concern. 2009. Only 1 in 6 older people embrace social networking and Age Concern wants more. Age Concern, London, UK, (March 2009). http://www.ageconcern.org.uk/AgeConcern/ social•networking-release-130309.asp

[2] Arjan, R., Pfeil, U., and Zaphiris, P. 2008. Age Differences in Online Social Networking. In Proc. CHI 2008, ACM Press, 2739-2744.

[3] Barrick, M.R. and Mount, M.K. 1991. The Big Five personality dimensions and job performance: $\mathrm{A}$ meta•analysis. Personal Psychology, 44 (1), 1-26.

[4] Braun, V. and Clarke, V. 2006. Using thematic analysis in psychology. Qualitative Research in Psychology, 3, 77-101.

[5] Churchill, E.F. and Halverson, C.A. 2005. Social networks and social networkings. IEEE Internet Computing, 9 (5), 14-19.

[6] Czaja, S. J., Guerrier, J. H., Nair, S. N., and Laudauer, T. K. 1993. Computer communication as an aid to independence to older adults. Behaviour and Information Technology , 12, 197-207.

[7] Dickinson, A., Smith, M., Arnott, J., Newell, A., and Hill, R. 2007. Approaches to web search and navigation for older computer novices. In Proc. $\mathrm{CHI}$ 2007, ACM Press, 281-290.

[8] Donath, J. 2004. Social media. In W.S. Bainbridge (Ed.), The encyclopedia of humancomputer interaction (1st ed.,). Massachusetts, USA: Berkshire Publishing Group.

[9] Ellison, N., Steinfield, C., and Lampe, C. 2006. Spatially Bounded Online Social Networks and Social Capital: The Role of Facebook. In Proc. Annual Conference of the International Communication Association.
[10] Ferlander, S. 2007. The importance of different forms of social capital for health. Acta Sociologica, 50, 115-128.

[11] Forbes, P., Gibson, L., Hanson, V.L., Gregor, P., and Newell, A.F. 2009. Dundee user centre: a space where older people and technology meet. In Proc. SIGACCESS (ASSETS '09), ACM Press, 231-232.

[12] Francis, D. 1991. Friends from the workplace. In Growing Old in America, B.B. Hess and E.W. Markson, Eds. Transaction Publishers, NJ, USA, 465-480.

[13] Glass, T. A., Matchar, D. B., Belyea, M., and Feussner, J. 1993. Impact of social support on outcome in first stroke. Stroke, 24, 64-70.

[14] Harley, D. and Fitzpatrick, G. $\quad 2 \quad 0 \quad 0 \quad 9$. YouTube and Intergenerational Communication: The case of Geriatric1927. Universal Access in the Information Society, 8(1), 5-20.

[15] Hill, R. A. and Dunbar, R.I.M.

20003.

Social network size in humans. Human Nature, 14(1), 53-72.

[16] Kraut, R., Kiesler, S., Boneva, B., Cummings, J., Helgeson, V., and Crawford, A. 2002. Internet Paradox Revisited. Journal of Social Issues, 58, 1, 49-74.

[17] Lehtinen, V., Näsänen, J., and Sarvas, R. 2009. "A Little Silly and Empty-Headed" - Older Adults' Understandings of Social Networking Sites. In Proc. HCl 2009 - People and Computers XXIII Celebrating people and technology, 45• 54 .

[18] Lindley, S. E., Harper, R., and Sellen, A.

2008. Designing for Elders: Exploring the Complexity of Relationships in Later Life. In Proc. $\mathrm{HCl} 2008-$ People and Computers XXIII - Culture, Creativity, Interaction, 77-86.

[19] Massimi, M. and Charise, A.

2009. Dying, death, and mortality: Towards thanatosensitivity in $\mathrm{HCl}$. In Proc. 27th International Conference Extended Abstracts on Human Factors in Computing Systems, Boston, MA, USA. 2459•2468. [20] Matthews, S. H. 1986. "Friendship Styles," Friendships Through the Life Course: Oral Biographies in Old Age. Sage Publication, 35-58.

[21] McLoone, P. 2004. Carstairs scores for Scottish postcode sectors from the 2001 Census. MRC Social \& Public Health Sciences Unit, Glasgow, Scotland. [22] Moncur, W., Reiter, E., Masthoff, J., and Carmichael, A. (2010). Modelling the socially intelligent communication of health information. IEEE Transactions on Information Technology in BioMedicine, 14, 2, 319-325.

[23] Morris, M.E. 2005. Social networks as health feedback displays. IEEE Internet Comput., 9, 5 (Sept/Oct 2005), 29・ 37.

[24] Ning. http://www.ning.com. Last accessed on 7th June 2010.

[25] OFCOM. 2009. The communications market report 2009. OFCOM, London, UK, (August 2009). http://www.ofcom.org.uk/research/cm/cmr09/ 
[26] Pfeil, U., Arjan, R., and Zaphiris, P. 2009.

Age differences in online social networking - A study of user profiles and the social capital divide among teenagers and older users in MySpace. Computers in Human Behavior, 25, 643-654.

[27] Please Rob Me. http://pleaserobme.com/. Last accessed on 7th June 2010.

[28] Quadrello, T., Hurme, H., Menzinger, J., Smith, P.K., Veisson, M., Vidal, S., and Westerback, S. 2005. Grandparents use of new communication technologies in a European perspective. European Journal of Ageing, 2, 200 207.

[29] Roberts, S.G.B., Wilson, R., Fedurek, P., and Dunbar, R.I.M. 2008. Individual differences and personal social network size and structure. Personality and Individual Differences, 44, 4 (March 2008), 954-964.

[30] SagaZone. http://www.sagazone.co.uk. Last accessed on 7th June 2010.

[31] Selwyn, N. 2004. The information aged: A qualitative study of older adults' use of information and communications technology. Journal of Aging Studies, 18, 369-384.

[32] Stiller, J. and Dunbar, RIM. 2007.

Perspective-taking and memory capacity predict social network size. Social Networks, 29(1), 93-104. [33] UN Programme on Ageing and the International Association of Gerontology and Geriatrics. 2007. Research agenda on ageing for the 21st century: 2007 Update. United Nations, New York, NY, USA, 2007. 